\title{
Pericardial effusion: The key point is the underlying etiology. Authors' reply
}

We would like to thank the authors for the letter [1] regarding our manuscript entitled "Prolonged Tp-e interval and Tp-e/QT correlates well with modified Rodnan skin severity score in patients with systemic sclerosis" [2] published in "Cardiology Journal". We would also like to thank the Editor for the opportunity to respond to this letter. In the study we aimed to evaluate Tp-e interval and Tp-e/QT ratio in patients with systemic sclerosis. We found that the patients with systemic sclerosis had a prolonged Tp-e interval and Tp-e/QT ratio compared with healthy subjects. Furthermore, this prolongation was well correlated with clinical severity score among patients with systemic sclerosis [2].

In the current letter, the authors questioned the relationship between pericardial effusion and QT dispersion. However, it is noteworthy, that pericardial effusion is not an entirely distinct disease, and the key point is to define the underlying etiology [3]. In this letter, the authors stated that "Pericardial effusion is well known to make changes in the electrocardiogram. Moreover, Yanagisawa et al. [4] found the relationship between pericardial effusion and QT dispersion. Also, Oliva et al. [5] found $T$ wave changes in patients with pericardial effusion." However, these two studies are somehow irrelevant with their comments.

In the first study, Yanagisawa et al. [4] noted that pericardial effusion is a potentially fatal com- plication following hematopoietic stem cell transplantation (HSCT). Common causes of pericardial effusion after HSCT were the reaction to allogeneic HSCT, the conditioning regimen, graft-versushost disease, infection, primary disease relapse, iron overload, and reaction to sirolimus or any immunosuppressant. In this analysis, Yanagisawa et al. [4] evaluated the efficacy of QT dispersion and corrected QT dispersion to predict pericardial effusion after HSCT. Their results suggest that pediatric patients with abnormally prolonged corrected QT dispersion before the preparative regimen for HSCT should be regularly followed-up by echocardiography to detect pericardial effusion, especially when accompanied by complications including transplantation-associated thrombotic microangiopathy. In the second study, reported 22 years ago, Oliva et al. [5] investigated the presence or absence of atypical $\mathrm{T}$ wave evolution in patients with post-infarction pericardial effusion. In this study, $\mathrm{T}$ wave alterations were observed when post-infarction pericardial effusion existed because of epicardial involvement in acute myocardial infarction. To clarify the specific question in the letter, we compared Tp-e interval and Tp-e/QT ratio between patients with pericardial effusion and without pericardial effusion. As expected, there was no significant difference in Tp-e interval and Tp-e/QT ratio between the two subgroups (see Figure 1).
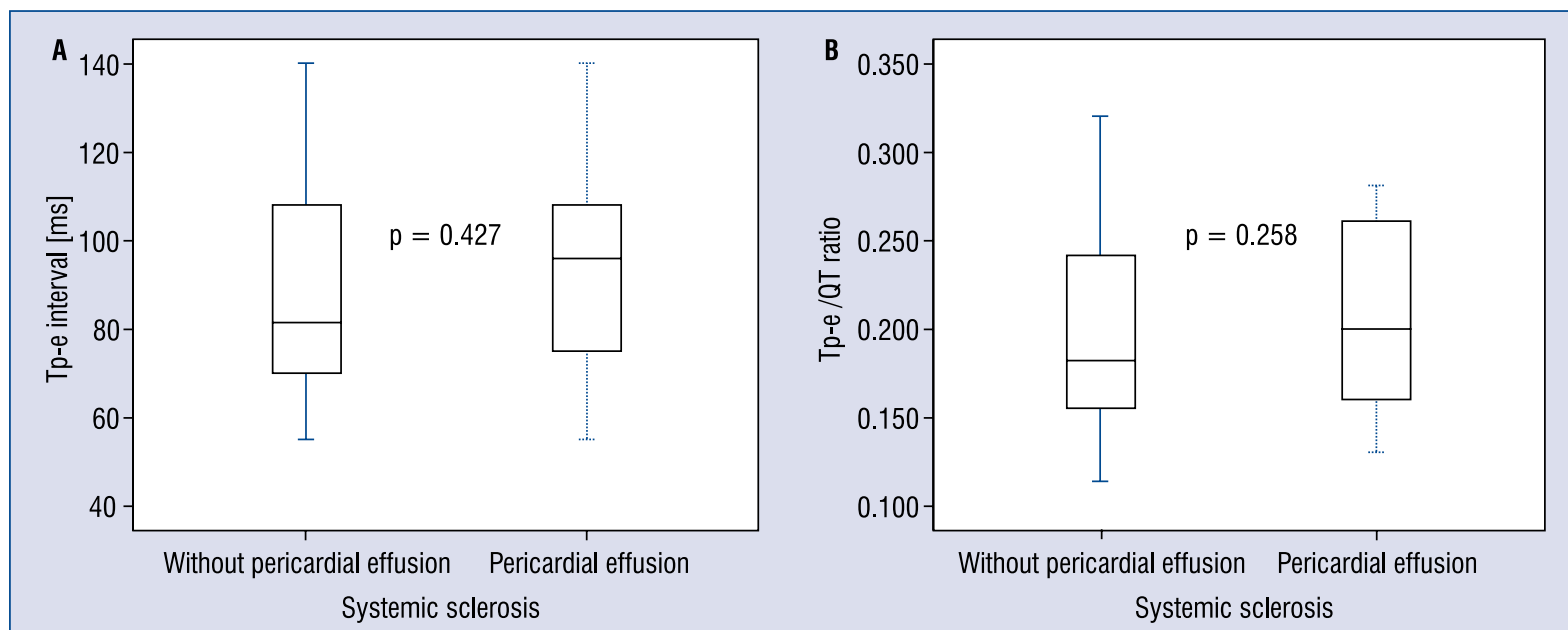

Figure 1. Tp-e interval (A) and Tp-e/QT ratio (B) in patients with systemic sclerosis with respect to presence of pericardial effusion. 
As a conclusion, pericardial effusion is a finding of everyday clinical practice. The main challenge is to establish the potential etiology. Sometimes, the pericardial effusion can be easily related to a known underlying disease, such as acute myocardial infarction, cardiac surgery, end-stage renal disease, or widespread metastatic neoplasm [3]. Therefore, electrocardiographic changes and myocardial repolarization abnormalities as well as structural alterations should be evaluated in the context of underlying disease.

Conflict of interest: None declared

\section{References}

1. Yayla Ç, Yayla KG, Ertem AG, Açar B, Ünal S, Akboğa MK. Pericardial effusion can affect the Tp-e interval and Tp-e/QT ratio. Cardiol J, 2016; 23: 360. doi: 10.5603/CJ.2016.0039.

2. Okutucu S, Karakulak U, Aksoy H et al. Prolonged Tp-e interval and Tp-e/QT correlates well with modified Rodnan skin severity score in patients with systemic sclerosis. Cardiol J, 2016; 23: 242-249. doi: 10.5603/CJ.a2016.0021.

3. Santas E, Nunez J. Prognostic implications of pericardial effusion: The importance of underlying etiology. Int J Cardiol, 2016; 202: 407.

4. Yanagisawa R, Ishii E, Motoki N et al. Pretransplant-corrected QT dispersion as a predictor of pericardial effusion after pediatric hematopoietic stem cell transplantation. Transpl Int, 2015; 28: 565-574.

5. Oliva PB, Hammill SC, Talano JV. T wave changes consistent with epicardial involvement in acute myocardial infarction. Observations in patients with a postinfarction pericardial effusion without clinically recognized postinfarction pericarditis. J Am Coll Cardiol, 1994; 24: 1073-1077.

\footnotetext{
Sercan Okutucu ${ }^{1}$, Ugur Nadir Karakulak ${ }^{2}$, Hakan Aksoy ${ }^{1}$, Ali Oto ${ }^{1}$

${ }^{1}$ Memorial Ankara Hospital, Department of Cardiology, Ankara, Turkey Cankaya/Ankara, P.O: 06520, tel: +90312 2536666 (ext. 4207), mobile: +90 5323314198 , fax: +90312 2536623,

e-mail:sercanokutucu@yahoo.com

${ }^{2}$ Occupational Diseases Hospital, Cardiology Department, Ankara, Turkey
} 